\title{
Thermal Expansion of Supported Thin Polymer Films: A Direct Comparison of Free Surface vs Total Confinement
}

\section{Darrin J . Pochan, ${ }^{*, t, \S}$ Eric K. Lin, ${ }^{\dagger}$ Sushil K. Satija, ${ }^{\ddagger}$ and Wen-li $\mathbf{W u}^{\dagger}$}

Polymers Division, NIST, Gaithersburg, Maryland, 20899; and NIST Center for Neutron Research, Gaithersburg, Maryland, 20899

Received J uly 18, 2000; Revised Manuscript Received J anuary 3, 2001

\begin{abstract}
Neutron reflectivity measurements on deuterated polystyrene (dPS) thin films reveal a strong dependence of the coefficient of thermal expansion (CTE) on both the thickness of the film and the specific confinement boundaries. A direct comparison is made between films on low energy, fluorinated polyimide (fPI) substrates with a free surface (bilayer) and the same films after capping the bilayer with another fPI layer (trilayer). For bilayers, a significant decrease in $\alpha_{r}$, the CTE above the bulk glass transition temperature, is observed in dPS with thicknesses $d<35 \mathrm{~nm}$. For trilayers, a decrease of $\alpha_{r}$ is observed at larger film thicknesses, $d<60 \mathrm{~nm}$. Replacing the fPI substrate with an $\mathrm{SiO}_{x}$ substrate, $\alpha_{r}$ remains bulklike, showing that the length scale for deviations from bulk behavior is sensitive to the specific confining boundaries.
\end{abstract}

\section{Introduction}

The integration of a wide variety of materials on the micro- and nanoscales is the physical foundation of the microelectronics industry. Knowl edge of many material properties, from dielectric constant to coefficient of thermal expansion, is critical for successful device design, simulation, and performance. Polymeric thin films, ubiquitous in el ectronics as packaging materials, are increasingly being investigated for use in active chip component applications and interlayer dielectrics. ${ }^{1}$

Much recent work has shown that polymers in confined geometries can have material properties different from those of the bulk material. F or example, the coefficient of thermal expansion of polymer thin films can be lower than that of the bulk material. ${ }^{2-4}$ More widely reported are changes in the apparent glass transition temperature, $T_{g}$, of supported thin polymer films with decreasing thickness. ${ }^{5-13}$ In the presence of favorable enthalpic interactions between the polymer film and the substrate, there is general experimental agreement that the apparent $\mathrm{T}_{\mathrm{g}}$ shifts to a higher temperature than observed in the bulk. However, less qualitative agreement is found when no strong enthal pic interactions are present between the polymer film and the confinement boundaries. Reflectivity experiments measure a rise in the apparent $T_{g},{ }^{6}$ optical and mechancial techniques measure a lowering of the apparent $\mathrm{T}_{\mathrm{g},}{ }^{11,13-15}$ and local dynamics techniques that monitor probe diffusion toward a film interface show no difference from bulklike behavior. ${ }^{16}$ Other thin film measurements suggest that minor differences in interfacial tension between polystyrene and substrate materials, such as silicon oxide vs hydrogen passivated silicon, produce starkly different film properties, particularly inducing either positive or negative shifts in $\mathrm{T}_{\mathrm{g}} .{ }^{6,14} \mathrm{The}$ free surface has also been identified as an important factor in the deviation of thin film properties from the

\footnotetext{
* To whom correspondence should be addressed.

† Polymers Division, NIST

₹ NIST Center for Neutron Research.

$\S$ Present address: Materials Science and Engineering Dept., University of Delaware, Newark, DE 19716.
}

bulk. More specifically, a reduced $T_{g}$ is postulated to exist in the first $2 \mathrm{~nm}$ at the surface layers and in freestanding films.2,17,18 The lack of agreement between different groups and techniques on similar systems suggests that subtle, but extremely important, experimental effects are yet to be identified. Only by understanding the combination, and possible synergism, of all possible enthalpic effects (polar/polar, hydrogen bonding, van der Waals) and entropic effects (molecular conformation perturbation, free surface effects) can one begin to comprehensively understand and predict polymer behavior in multimaterial, highly interfacial environments like those encountered in microelectronics devices.

In this article we report the first direct comparison of free surface vs rigid confinement boundary conditions (substrate supported film with no superstrate vs film confined between both a sub- and superstrate) on the thermal expansion behavior of the same deuterated polystyrene (dPS) thin films. ${ }^{19}$ Neutron reflectivity (NR) measurements are used to determine the thickness of thin $(<100 \mathrm{~nm})$, amorphous polymer films as a function of temperature. To focus on the effects of confining polymer molecules in a thin film geometry, we eliminated specific energetic interactions between the substrate and the polymer film by using a fluorinated polyimide ( $\mathrm{fPI})^{20,21}$ as the confining boundary. The fPI layer provides a rigid, low surface energy, impenetrable interface that subjects the dPS layer to weak, long range, dispersive van der Waals forces. To facilitate comparisons with other work, the thermal expansi on of dPS films with the fPI substrate replaced by the native oxide surface of the supporting silicon wafer was also measured.

\section{Experimental Section}

To prepare the samples, thin layers of fPI (bulk $T_{g}=300$ $\left.{ }^{\circ} \mathrm{C}\right) 50 \mathrm{~nm}$ thick were first spin-coated from cyclopentanone solutions onto extensively cleaned (111) single-crystal silicon wafers. The wafers were $76.2 \mathrm{~mm}$ in diameter, $5 \mathrm{~mm}$ thick, and prepared with an oxide layer, $\mathrm{SiO}_{x_{1}} \approx 2.0 \mathrm{~nm}$ thick. ${ }^{26}$ The fPI layers were then anneal ed at $250^{\circ} \mathrm{C}$ under vacuum $\left(<10^{-5}\right.$ Torr) for $12 \mathrm{~h}$. Bilayer samples were prepared by floating a spin-coated monodisperse dPS $\left(\mathrm{M}_{\mathrm{w}}=260000,{ }^{22}\right.$ bulk $\mathrm{T}_{\mathrm{g}}=100$ 




Figure 1. Neutron reflectivity data from both an fPI/dPS bilayer and the subsequently capped fPI/dPS/fPI trilayer samples supported on a silicon wafer. The dPS film thickness is $70 \mathrm{~nm}$. The points are the experimental data and the solid lines are the best fits to the data. The experimental error bars are generally within the size of the points. The real space profiles corresponding to best fits to the experimental data are shown as insets.

$\left.{ }^{\circ} \mathrm{C}\right)$ layer onto ultrapure water and transferring it onto the fPI-coated silicon substrate. The bilayer samples were then anneal ed at $150{ }^{\circ} \mathrm{C}$ under vacuum for $12 \mathrm{~h}$ and cooled to room temperature over a period of several hours. After NR measurements, trilayer samples were prepared using the floating method to cap the bilayer samples with another layer of fPI $\approx 150 \mathrm{~nm}$ thick, thus creating samples with a $\mathrm{SiO} \times \mathrm{fPI} / \mathrm{dPS} /$ $\mathrm{fPI}$ geometry. Trilayers were further annealed at $150^{\circ} \mathrm{C}$ under vacuum for $12 \mathrm{~h}$ and cool ed to room temperature over several hours. NR measurements were performed again, al lowing the observation of the same dPS layers in both the bilayer and trilayer geometries. For comparison of different confinement materials, dPS was also spin cast directly onto $\mathrm{SiO}_{x}$ and anneal ed at $150{ }^{\circ} \mathrm{C}$ ) to produce $\mathrm{SiO}_{x} / \mathrm{dPS}$ bilayer samples. In addition after NR measurements, $\mathrm{SiO}_{x} / \mathrm{dPS} / \mathrm{fPI}$ trilayers were made via the floating and annealing procedure above and subsequently measured via NR. No observable dewetting or morphological changes occurred in any samples during any of the annealing steps or during the thermal expansion measurements.

The NR measurements were performed on the National Institute of Standards and Technology Center for Neutron Research NG7 reflectometer in a specially designed high vacuum chamber. The wavel ength, $\lambda$, was $4.768 \AA$ with $\Delta \lambda / \lambda$ $=0.20$. B efore each NR measurement, the samples were raised to a set temperature over approximately $15 \mathrm{~min}$ and held at the set temperature $\left( \pm 0.2{ }^{\circ} \mathrm{C}\right)$ for at least $30 \mathrm{~min}$. The reflectivity data are presented as a function of the scattering vector in the direction perpendicular to the polymer film plane, $\mathrm{Q}=(4 \pi / \lambda) \sin \theta)$, where $\theta$ is the incident and detected angle. The reflectivity data are modeled and fit with a nonlinear, least-squares method using the recursive multilayer method. ${ }^{23}$ Figure 1 displays two representative experimental neutron reflectivity curves with the best model fits to the data for a bilayer and trilayer sample at room temperature. The corresponding scattering length density profiles are shown in the inset. The simple sample geometry permits a clear determination of dPS thickness with additional sensitivity to experimental concerns such as interfacial broadening, density gradients, and impurities at interfaces. ${ }^{24,25}$ The measured interfacial roughness between the fPI boundaries and the dPS layer in both bilayer and trilayer samples ranges from 0.5 to $1.5 \mathrm{~nm}$ and remains constant throughout the experiment. F or the remainder of this article, the error bars in each figure represent the standard uncertainty for each point. Several of the bilayer and thinnest trilayer samples were also measured with X-ray reflectometry. No hysteresis in the determined thickness was observed between heating and cooling runs, and the dPS thickness at each temperature was within the standard uncertainty of the NR determined thickness. In the



Figure 2. Normalized dPS film thickness as a function of temperature, $d_{T} / d_{0}$ where $d_{T}$ is the film thickness at temperature and $\mathrm{d}_{\mathrm{O}}$ is the initial dPS film thickness, for four samples with initial dPS thicknesses (a) 70, (b) 60, (c) 35, and (d) 15 $\mathrm{nm}$. The bilayer data are given by the solid symbols, and the trilayer data are shown by the open symbols. The solid lines represent the thermal expansion curves of bulk PS. The data for each set are vertically offset for clarity. A schematic of the bilayer and trilayer sample geometry is shown above the thickness plot.

trilayer samples, the scattering length density of the fPI superstrate is $20 \%$ lower than the substrate fPI layer. Annealing close to the bulk $\mathrm{T}_{\mathrm{g}}\left(300^{\circ} \mathrm{C}\right)$ of the fPI was not done in the presence of the dPS to prevent any possible interdiffusion.

\section{Results and Discussion}

Figure 2 shows plots of the normalized dPS film thickness vs temperature for 4 different dPS films (a) 70, (b) 60, (c) 35, and (d) $15 \mathrm{~nm}$ thick in both the bilayer (filled symbols) and trilayer (open symbols) geometries. The solid lines represent the bulk thermal expansion behavior of dPS glass $\left(\alpha_{\mathrm{g}}\right)$ and rubber (liquid) $\left(\alpha_{\mathrm{r}}\right)$ below and above the $\mathrm{T}_{\mathrm{g}}$ of $100{ }^{\circ} \mathrm{C}$. The bulk linear coefficients of thermal expansion, $\alpha_{1}$, have been corrected for the constraint that a polymer film in contact with a rigid substrate expands only in the direction normal to the sample plane. We use Poisson's ratio, $v$, for bulk $\mathrm{dPS}^{6}$ via the relationship $\alpha_{1}[(1+\mathrm{v}) /(1-\mathrm{v})]$ where $v=0.325$ and $\alpha_{1}=0.57 \times 10^{-4} /{ }^{\circ} \mathrm{C}$ at $\mathrm{T}<\mathrm{T}_{\mathrm{g}}$ and $v=0.5$ and $\alpha_{1}=$ $1.7 \times 10^{-4} /{ }^{\circ} \mathrm{C}$ at $\mathrm{T}>\mathrm{T}_{\mathrm{g}}$. This produces $\alpha_{\mathrm{g}}=1.1 \times 10^{-4} /$ ${ }^{\circ} \mathrm{C}$ at $\mathrm{T}<\mathrm{T}_{\mathrm{g}}$ and $\alpha_{\mathrm{r}}=5.1 \times 10^{-4} /{ }^{\circ} \mathrm{C}$ at $\mathrm{T}>\mathrm{T}_{\mathrm{g}}$. To make meaningful observations, we compare measured thin film properties with the properties observed in the bulk state. This comparison for all properties best defines and characterizes any and all confinement effects of the thin film geometry.

The validity of this bulk behavior comparison is demonstrated in Figure 2, parts $a$ and $b$. The CTE and $\mathrm{T}_{\mathrm{g}}$ of the two thickest bilayer samples of 70 and $60 \mathrm{~nm}$ dPS are consistent with bulklike behavior. Although not shown here, several other thicker dPS films also exhibited bulklike thermomechanical behavior in both the bilayer and trilayer geometries. Also consistent with 
other measurements, the glassy thermal expansion remains essentially constant for all samples studied here. However, the $\alpha_{r}$ values dramatically decrease as the films decrease in thickness for both bilayers and trilayers. Considering the set of bilayer samples, $\alpha_{r}$ drops from the bulklike value to $(2.5 \pm 0.7) \times 10^{-4} /{ }^{\circ} \mathrm{C}$ at $\mathrm{T}>100^{\circ} \mathrm{C}$ when the dPS thickness is reduced to 35 $\mathrm{nm}$ in Figure $2 \mathrm{c}$. For the thinnest sample, Figure $2 \mathrm{~d}$, no apparent $T_{g}$ is observed and the bulklike $\alpha_{g}$ persists throughout the temperature range. For the trilayer samples, $\alpha_{r}$ is consistently reduced relative to the corresponding bilayer (Figure 2a-2c). The bulklike $\alpha_{r}$ of $(5.9 \pm 0.6) \times 10^{-4} /{ }^{\circ} \mathrm{C}$ at $\mathrm{T}>100{ }^{\circ} \mathrm{C}$ in $2 a$ has dropped to $(4.0 \pm 0.5) \times 10^{-4} /{ }^{\circ} \mathrm{C}$ while $\alpha_{g}$ persists throughout the temperature range observed in Figure $2 b-d$. A break in slope may occur at $\approx 150{ }^{\circ} \mathrm{C}$ in Figure $2 \mathrm{~d}$ al though not enough data points are present above 150 ${ }^{\circ} \mathrm{C}$ to be conclusive. The changes in the thermal expansion between the bilayer and trilayer cases, particularly for the $60 \mathrm{~nm}$ film, are remarkable because the only difference is the replacement of a vacuum boundary with an fPI one.

From the data in Figure 2, there appears to be an interface induced, characteristic pertubative lengthscale for $\alpha_{r}$. When the dPS film thickness drops below this value the thermomechanical properties are affected. The perturbation length goes from virtually zero in fPI substrate/free surface superstrate pair to $60 \mathrm{~nm}$ after exchanging the free surface boundary with an fPI confinement layer. A free surface provides a much different confinement boundary than the fPI confinement material despite the fact that both are energetically noninteractive, or even repulsive, to dPS. A coupling effect between both fPI interfaces must be present to induce such a drastic change in perturbation length of the dPS film properties. This perturbation length is also strongly dependent upon the specific substrate and superstrate pairing. This is clear from a comparison of the thermal expansion data in Figure 2, parts $\mathrm{c}$ and $\mathrm{d}$, with films of similar dPS thickness in Figure 3, parts a and b. In Figure 3, the "bilayer" is a dPS film floated onto the native $\mathrm{SiO}_{x}$ and the corresponding "trilayer" is in a $\mathrm{SiO}_{x} / \mathrm{dPS} / \mathrm{fPI}$ configuration. In stark contrast to the fPI/dPS/free surface bilayers, the $\mathrm{SiO}_{x} / \mathrm{dPS} /$ free surface bilayers exhibit bulklike thermomechanical properties even for the thinnest films studied. After further confinement with a superstrate of fPI, bulk behavior is still exhibited. The approximately bulk CTE values for these films is consistent with other reported CTE values of PS on $\mathrm{SiO}_{x^{*}}{ }^{2,7}$ There is clearly a marked difference between the free surface and the solid $\mathrm{SiO}_{x}$ interface as a confinement boundary and their respective coupling with the fPI layer.

The strong dependence of polymer thin film material properties on both thickness and state of confinement (bilayer vs trilayer) is often analyzed in terms of a multilayer model. In such a model, the dPS film consists of a layer exhibiting bulk properties between a persistently glassy layer next to the substrate and/or a more mobile layer at the polymer free surface. For example, higher $\mathrm{T}_{\mathrm{g}}$ and slower polymer dynamics in thin films have been attributed to specific interactions between polar polymer segments (such as poly(methyl methacrylate) and poly(vinylpyridine) and the substrate material $\left(\mathrm{SiO}_{\mathrm{x}}\right)^{3,4,26}$ These favorable interactions are thought to produce physical cross-links between the polymer segments and the substrate that effectively pin



Figure 3. Normalized dPS film thickness as a function of temperature for two samples with initial dPS thicknesses of (a) 24 and (b) $45 \mathrm{~nm}$. The filled symbols are data from SiO dPS bilayers, and the open symbols are data from $\mathrm{SiO}_{x} / \mathrm{dPS} /$ fPI trilayers with the same dPS film. The data sets from the two samples are vertically offset for clarity.

the chains at the interface introducing an persistently glassy, "dead" polymer layer at the substrate/polymer interface. Decreases in $\alpha_{r}$ have been interpreted with similar dead layer arguments where as the overall film thickness decreases, the pinned interfacial layer begins to dominate the thermal expansion. 2,4,6 Alternatively, apparent $T_{g}$ shifts to lower temperatures with decreasing film thickess have prompted propositions of a liquidlike layer of constant thickness at the air/polymer interface. 2,14 In the present study, the fluorinated substrate and superstrate layers provide for low energy surfaces with no favorable, specific interactions with the dPS layer.

In considering a stratification of different material properties in dPS, let us first address trends in the bilayer data relative to thickness. The two thickest films exhibit bulklike thermal expansion behavior. However, moving from 60 to $35 \mathrm{~nm}$ (Figure $2 \mathrm{~b}$ to $2 \mathrm{c}$ ) a marked decrease in $\alpha_{r}$ is easily observed. While $\alpha_{g}$ remains bulklike for $\mathrm{T}<100^{\circ} \mathrm{C}, \alpha_{r}$ drops to $(2.5 \pm 0.7) \times 10^{-4} /$ ${ }^{\circ} \mathrm{C}$ from the theoretical bulk value of $5.1 \times 10^{-4} /{ }^{\circ} \mathrm{C}$. Assuming polymer confinement at the solid substrate interface produces a two-tiered material, one with a dead layer exhibiting $\alpha_{g}$ throughout the temperature range observed and the remainder exhi biting bulk CTE properties, and further assuming that the contributions from the bulk stratus and the dead-layer are additive, a dead-layer thickness of $24 \mathrm{~nm}$ is calculated for the 35 $\mathrm{nm}$ film. The thinnest film in Figure $2 \mathrm{~d}$ is consistent with this, exhibiting glasslike expansion throughout the temperature range studied since the entire film thickness of $15 \mathrm{~nm}$ is less than the dead layer thickness of $24 \mathrm{~nm}$. However, in considering all four bilayer plots, the multilayer dPS analysis is not consistent. A persistently glassy $24 \mathrm{~nm}$ interfacial layer would reduce $\alpha_{r}$ at $\mathrm{T}>100{ }^{\circ} \mathrm{C}$ in Figure 2, parts $\mathrm{a}$ and $\mathrm{b}$, by $25 \%$ and $27 \%$ respectively, well outside the experimental uncertainty in measured thickness change. A trilayer model 
(three layers within the dPS layer-not to be confused with the fPI/dPS/fPI trilayer geometry described earlier), consisting of an enhanced rubbery regime at the polymer/air interface at $T<\mathrm{T}_{\text {g,bulk }}$ that counteracts the persistently glassy behavior induced by substrate confinement, has been proposed to explain thin film properties. ${ }^{2}$ This is not a reasonable explanation of the bilayer data for two reasons. First, the reported depths to which the free surface affects polymer properties are limited to $2 \mathrm{~nm}$. Top layers with enhanced mobility of this thickness range would require an unphysical CTE of $\approx 6 \times 10^{-4}$ to $9 \times 10^{-4} /{ }^{\circ} \mathrm{C}$ in order to counteract a 24 $\mathrm{nm}$ dead layer and yield the measured bulk CTE. Second, this polymer/air interfacial layer would most likely exhibit a lower $\mathrm{T}_{\mathrm{g}}$ than the bulk polymer presumably due to a reduced density. H owever, these ideas are contradicted by both the bulklike apparent $\mathrm{T}_{\mathrm{g}}$ and the $\alpha_{g, \text { bulk }}$ observed for all films at $\mathrm{T}<100{ }^{\circ} \mathrm{C}$.

This type of multilayer analysis also fails when comparing the bilayer and trilayer geometries. After capping the film in Figure $2 a$, we observe an $\alpha_{r}$ of $(4.0$ $\pm 0.5) \times 10^{-4} /{ }^{\circ} \mathrm{C}$ at $\mathrm{T}>100{ }^{\circ} \mathrm{C}$. This corresponds to a dPS dead layer of total thickness $31 \mathrm{~nm}$. According to the previous determination in the bilayer sample of a $24 \mathrm{~nm}$ dead layer, confinement-altered layers at both the top and bottom dPS film interface would produce a total dead-layer thickness of $48 \mathrm{~nm}$, not $31 \mathrm{~nm}$, and an $\alpha_{r}$ of $2.5 \times 10^{-4} /{ }^{\circ} \mathrm{C}$, a $37 \%$ deviation from the observed $\alpha_{r}$. Interestingly, the hypothetical sub- and superstrate confinement effect appears at $\mathrm{d}=60 \mathrm{~nm}$ in the trilayer of Figure $2 \mathrm{~b}$ with $\alpha_{g}$ persisting throughout the temperature range observed. However, the dead layer is not present in the $60 \mathrm{~nm}$ bilayer data in Figure 2b. Not surprisingly, the trilayer data in Figure 2, parts $\mathrm{c}$ and $d$, exhibit $\alpha_{\text {g,bulk }}$ expansion throughout the temperature range studied. From the discussion above, it is clear that a simple multilayer model does not account for the property changes occurring in the dPS thin films presented here.

Other arguments have been proposed to explain deviations in either CTE and/or $\mathrm{T}_{\mathrm{g}}$ behavior from the bulk behavior. Assume the persistence of $\alpha_{g}$ well above the bulk $\mathrm{T}_{\mathrm{g}}$ of $100{ }^{\circ} \mathrm{C}$ in Figure 2 is indicative of $\mathrm{T}_{\mathrm{g}}$ rising to higher temperatures. While long-range, dispersive van der Waals forces have recently been shown to have a marked effect on both the stability of supported, thin polymer films ${ }^{27}$ and morphology of freely standing polymer films, ${ }^{28}$ these forces are much too weak to induce effective $T_{g}$ changes that seem to occur in the thin films. In a simple calculation in which the polyimide layers are treated as highly fluorinated infinite planes, the Hamaker constant, A, is estimated to be A $=3.6 \times 10^{-21} \mathrm{~J}$ for dPS between fPI layers. ${ }^{29}$ The relation $\mathrm{P}=\mathrm{A} / 6 \pi \mathrm{d}^{3}$ can be used to estimate $1-1000 \mathrm{~Pa}$ of compressive pressure on the sandwiched dPS layer for dPS film thicknesses of 50-5 nm, respectively. ${ }^{8}$ The disjoining pressure experienced by the dPS film in the $\mathrm{fPI} / \mathrm{dPS}$ bilayer with a free surface is of the same order of magnitude, several orders of magnitude too small to produce even $1{ }^{\circ} \mathrm{C}$ of change in $\mathrm{T}_{\mathrm{g}}$ given the bulk empirical relationship $\partial \mathrm{T}_{\mathrm{g}} / \partial \mathrm{P}=3.09 \times 10^{-7}{ }^{\circ} \mathrm{C} / \mathrm{Pa}{ }^{30}$ Also, a change in the average film density with shrinking film thickness, or an overall thickness dependence of the polymer film density, has been suggested as possible reasons for a change in $T_{g} / C T E$ of thin polymer films, particularly for seemingly altered material properties very close (within 1-5 nm) to the air/polymer interface ${ }^{2,31} \mathrm{U}$ sing the relationship $\left(\partial \mathrm{T}_{\mathrm{g}} / \partial \rho\right)=\left(\partial \mathrm{T}_{\mathrm{g}} / \partial \mathrm{P}\right)$ $(\partial \mathrm{P} / \partial \rho)=(\partial \mathrm{T} / \partial \mathrm{P})(1 / \rho \kappa)$, where $\kappa$ is the isothermal compressibility $\kappa=2.2 \times 10^{-10} \mathrm{~Pa}^{-1}, 32$ a density increase of $1.25-2.50 \%$ over bulk values is required for a $20-40{ }^{\circ} \mathrm{C}$ rise in $\mathrm{T}$. This shift is improbable, especially in light of recent measurements of bulk average densities to within $1.0 \%{ }^{33,34}$ and, in the case of neutron interferometry measurements, ${ }^{35}$ within $0.5 \%$ of the bulk value for polystyrene thin films.

\section{Conclusions}

The complexity of the CTE data reveals an important characteristic of the polymer thin films: the material properties are altered within a characteristic perturbation distance of an interface that is dependent on (a) the overall dPS film thickness and (b) the particular substrate/superstrate pairing. The thickness dependence is revealed within the bilayer and trilayer sets of data. Bilayer samples below $40 \mathrm{~nm}$ in dPS thickness suggest an interface-induced persistently glassy layer of, at most, $24 \mathrm{~nm}$. However, in the slightly thicker bilayer films of 60 and $70 \mathrm{~nm}$, a persistently glassy layer of this magnitude is not observed. Likewise, the trilayer samples with $d<60 \mathrm{~nm}$ suggest a persistently glassy layer at least $60 \mathrm{~nm}$ (30 nm from both dPS/fPI interfaces). However, the slightly thicker dPS trilayer film of $71 \mathrm{~nm}$ exhibits a persistently glassy layer thickness of merely $31 \mathrm{~nm}$ combined at the dPS/fPI interfaces. A strong coupling appears to exist between substrate and superstrate that influences the physical properties of the film. The marked ability of the low surface energy fPI to lower $\alpha_{r}$ above the bulk $T_{g}$ is clear and strongly suggests additional parameters need to be considered for predictions of polymer thin film behavior. In particular, one must consider the substrate/superstrate pair in attempts to characterize and understand the material properties of the polymer thin films.

Acknowledgment. The authors would like to thank Professor Stephen Z. D. Cheng of the University of Akron for providing the fPI materials. D.J .P. thanks the National Research Council and the National Institute of Standards and Technol ogy for postdoctoral fellowship support.

\section{References and Notes}

(1) Drury, C. I .; Mutsaers, C. M. I ; Hart, C. M.; Matters, M.; de Leeuw, D. M. Appl. Phys. Lett. 1998, 73, 108.

(2) DeMaggio, G. B.; Frieze, W. E.; Gidley, D. W.; Zhu, M.; Hristov, H. A.; Yee, A. F. Phys. Rev. Lett. 1997, 78, 1524.

(3) Wu, W. L.; van Zantan, J. H.; Orts, W. J . Macromolecules 1995, 28, 771.

(4) van Zanten, J. H.; Wallace, W. E.; Wu, W. L. Phys. Rev. E 1996, 53, R2053.

(5) Reiter, G. Macromolecules 1994, 27, 3046

(6) Wallace, W. E.; van Zanten, J. H.; Wu, W. L. Phys. Rev. E 1995, 52, R3329.

(7) Orts, W. J .; van Zanten, J . H.; Wu, W. L.; Satija, S. K. Phys. Rev. Lett. 1993, 71, 867.

(8) Keddie, J . L.; J ones, R. A. L.; Cory, R. A. Faraday Discuss., Chem. Soc. 1994, 98, 219.

(9) Forrest, J . A.; Dalnoki-Veress, K.; Dutcher, J . R. Phys. Rev. E 1997, 56, 5705

(10) Aubouy, M. Phys. Rev. E 1997, 56, 3370.

(11) Kleideiter, G.; Prucker, O.; Bock, H.; Frank, C. W.; Lechner M. D.; Knoll, W. Macromol. Symp. 1999, 145, 95

(12) See, Y. K.; Cha, J .; Chang, T.; Ree, M. Langmuir 2000, 16, 2351.

(13) Forrest, J . A.; Svanberg, C.; Revesz, K.; Rodahl, M.; Torell, L. M.; Kasemo, B. Phys. Rev. E 1998, 58, R1226.

(14) Keddie, J . L.; J ones, R. A. L.; Cory, R. A. Europhys. Lett. 1994 $27,59$. 
(15) Prucker, O.; Christian, S.; Bock, H.; Ruehe, J .; Frank, C. W.; Knoll, W. Macromol. Chem. Phys. 1998, 199, 1435

(16) Hall, D. B.; Torkelson, J. M. Macromolecules 1998, 31, 8817.

(17) Kajiyama, T.; Tanaka, K.; Satomi, N.; Takahara, A. Macromolecules 1998, 31, 5150 .

(18) Forrest, J . A.; Dalnoki-Veress, K.; Stevens, J . R.; Dutcher, J . R. Phys. Rev. Lett. 1996, 77, 2002.

(19) In ref 10, Forrest et al. performed ellipsometry measurements on uncapped $\mathrm{SiO}_{x} / \mathrm{PS}$ bilayers and separate samples with $\left(\mathrm{SiO}_{x} / \mathrm{PS}\right)_{n}$ multilayer geometry in an attempt to observe changes in $\mathrm{T}_{\mathrm{g}}$ of PS with confinement. However, different samples at approximately the same thickness were used in the two different geometries with thin, $7 \mathrm{~nm}$ evaporated $\mathrm{SiO}_{x}$ layers used as the confinement layers, a much different chemical environment than the thicker, high $\mathrm{T}_{\mathrm{g}}$, fPI confinement layers used in this study.

(20) Kwan, S. C. M.; Wu, C.; Li, F.; Savitski, E. P.; Harris, F. W.; Cheng, S. Z. D. Macromol. Chem. Phys. 1997, 198, 3605.

(21) Li, F.; Kim, K. H.; Savitski, E. P.; Chen, J . C.; Harris, F. W.; Cheng, S. Z. D. Polymer 1997, 38, 3223.

(22) According to ISO 31-8, the term "M olecular Weight" has been replaced by "Relative Molecular Mass," symbol $\mathrm{M}_{\mathrm{r}}$. Thus, if this nomenclature and notation were to be followed in this publication, one would write $M_{r}$, instead of the historically conventional $\mathrm{M}_{\mathrm{n}}$ for the number average molecular weight, with similar changes for $M_{w}, M_{z}$, and $M_{v}$, and it would be called the "Number Average Relative Molecular Mass." The conventional notation, rather than the ISO notation, has been employed for this publication.

(23) Ankner, J. F.; Majkrzak, C. J . In Neutron Optical Devices and Applications; SPIE Proceedings 1738; SPIE: Bellingham, WA, 1992; p 260

(24) Russell, T. P. Mater. Sci. Rep. 1990, 5, 171

(25) Higgins, J. S.; Benoit, H. C. Polymers and Neutron Scattering; Clarendon Press: Oxford, England, 1994; Chapter 10.

(26) Lin, E. K.; Kolb, R.; Satija, S. K.; Wu, W. L. Macromolecules 1999, 32, 3753

(27) Reiter, G.; Sharma, A.; Casoli, A.; David, M. O.; Khanna, R.; Auroy, P. Langmuir 1999, 15, 2551.

(28) Dalnoki-Veress, K.; Nickel, B. G.; Dutcher, J . R. Phys. Rev. Lett. 1999, 82, 14861999.

(29) Israelachvili, J . N. I ntermol ecular and Surface Forces; Academic Press: New York, 1992; Chapter 11.

(30) Eisenberg, A. J . Phys. Chem. 1963, 67, 1333.

(31) Satomi, N.; Takahara, A.; Kajiyama, T. Macromol ecules 1999, $32,4474$.

(32) Polymer Handbook, 2nd ed.; Brandrup, J ., I mmergut, E. H., Eds.; J ohn Wiley and Sons: New York, 1975.

(33) Forrest, J . A.; Dalnoki-Veress, K.; Dutcher, J . R. Phys. Rev. E 1998, 58, 6109.

(34) Wallace, W. E.; Wu, W. L. Appl. Phys. Lett. 1995, 67, 1203.

(35) Wallace, W. E.; J acobsen, D. L.; Arif, M.; I offe, A. Appl. Phys. Lett. 1999, 74, 469.

MA001253F 